\title{
FOLLOW THE YELLOW BRICK ROAD
}

\author{
Tracing the routes that led to successful drugs can reveal a winding path, very different from \\ the linear track we like to depict. Are the models for drug development becoming prescriptive \\ rather than descriptive?
}

In mid-March, the Society for Medicines Research will be hosting a one-day meeting in London that will attempt to address the thorny issue of whether there is a best strategy for drug discovery? Rather a bold question for such a short meeting, certainly, but, viewed against the backdrop of the hundreds of meetings held around the world each year, which split the question of drug-discovery strategies into smaller, more digestible chunks, it offers a rare opportunity to take an overview of the discipline of drug discovery in its entirety. What might the assembled collection of proven champions, current standard bearers and upcoming reinforcements conclude about the way the battle should be waged in future years?

For starters, they are likely to agree that the story of every past victory in drug discovery is different. Each drug in existence today made it through to market in more or less its own way. Although this is widely recognized within the industry, and everyone can cite examples of drugs that did not follow the 'standard' pattern of drug development, it seems strange that we do not acknowledge this when describing current research strategies. Instead, we talk about a linear process in which targets, and then compounds, are fed from one stage to the next. Just think how many times we have reproduced 'that' diagram, with arrows joining each step in the serial sequence, in this journal. Another standardly used image of the drug-discovery process is the funnel, into which handfuls of compounds are dumped at the top, filtering down to a single, pristine drug-like compound at the bottom. Of course, these are partially true pictures, but ask any team that has successfully developed a drug to describe the path they took, and they draw a much more circuitous route. The 'standard' pattern is hard to find.

Despite knowing this, we still have the propensity to fall back on these simplified descriptions, which might be encouraging a new generation of potential drug discoverers to view the process as more sequential than it really is. Perhaps drug discovery and development will prove amenable to industrialization in the future, and production lines will churn merrily through the steps and spit drugs out at the end. Until this becomes the case, should we not be a little more circumspect when reaching for those favourite models, lest we mislead ourselves into believing that we are already there?

So when looking for the best strategy, one has to search for the common themes running through the success stories we have so far. Tracing the true story behind each drug can be a hard task. Seen with hindsight, most journeys look considerably smoother than they did at the time, and the crucial decisions of the past can be hard to pinpoint. This is why we instituted our series of Case History articles, which are personal views of the development of a particular drug or class of therapeutics, written by the people who were actually there at the time. "Tell it like it was", we ask our authors. "Don't pull the punches".

This issue features two such articles: Craig Jordan's description of the long road to Tamoxifen, and Rod Flower's account of the development of Vioxx and Celebrex. Both reveal key features of these histories that are at odds with the industrial view of drug development. In Tamoxifen's case, a drug first developed as a potential contraceptive languished for many years before its present application was found. Furthermore, its propensity to cause liver tumours in rats, a toxicity problem that thankfully does not carry over into humans, was not detected until after the drug had been on the market for many years. If it had been found in preclinical testing, the drug would almost certainly have been withdrawn from the pipeline. With the COX2 inhibitors, Rod Flower notes that the transgenic animal models used to test the hypothesis that COX2 would make an anti-inflammatory target gave results that, if relied upon, might have killed the project. Both stories emphasize the role of the dogged researchers who kept their eyes focused on the prize while navigating around obstacles and exploiting any opportunity that came along. It might sound a bit trite, but at the very least we can say that one of the best strategies for drug discovery is to start with a group of people who really want to discover drugs. 